\title{
Expression of Fatty Acid Synthase Negatively Correlates with PTEN and Predicts Peritoneal Dissemination of Human Gastric Cancer
}

\author{
Hong-Gang Xiang ${ }^{1 \&}$, Jun Hao ${ }^{2 \&}$, Wen-Jie Zhang ${ }^{1}$, Wen-Jie Lu' ${ }^{1}$, Ping Dong ${ }^{1}$, \\ Ying-Bin Liu ${ }^{1}$, Lei Chen ${ }^{1 *}$
}

\begin{abstract}
Background: This study aimed to examine the clinical significance of fatty acid synthase (FASN) expression in gastric cancer (GC), and investigate any prognostic role. Materials and Methods: FASN expression was assessed in gastric cancers by immunohistochemistry using 60 paraffin-embedded tissue specimens, and clinical data were collected by retrospective chart review. Moreover, FASN mRNA expression in 15 fresh resected specimens was evaluated by the reverse transcription-polymerase chain reaction (RT-PCR). Immunohistochemical staining of PTEN was performed to assess the correlation of PTEN with FASN in gastric cancer. Results: Increased expression of FASN was noted in gastric cancers. The frequency of FASN gene amplification was also significantly higher in gastric cancer than in adjacent normal tissue. FASN expression in human gastric cancer tissues was significantly correlated with patient TNM stage and peritoneal dissemination $(p<0.05)$. Moreover, higher FASN expression significantly correlated with shorter overall survival $(p<0.05)$. Here, upregulation of FASN negatively correlated with PTEN expression in gastric cancer. Conclusions: These findings indicate that FASN expression is upregulated in gastric cancer, and increased FASN may be critical to th peritoneal metastasis and survival. Our results suggest that FASN upregulation and PTEN downregualtion may be involved in peritoneal dissemination for gastric cancer progression.
\end{abstract}

Keywords: Gastric cancer - FASN - progression - PTEN - prognosis

Asian Pac J Cancer Prev, 16 (16), 6851-6855

\section{Introduction}

Gastric cancer is the one of most prevalent common malignant tumors and the common cause of cancer related death in East Asia. Gastric cancer is associated with a short time to recurrence and a short survival period after recurrence. In China, age-standardized 5-year relative survival for gastric cancer was only $27.4 \%$ (Zeng et al., 2014). Although advances in diagnostic and treatment technologies have offered long-term survival results for early gastric cancer, but the prognosis for advanced GC still remains poor. Advanced gastric cancer patients prone to progress to peritoneal carcinomatosis and the prognosis for gastric carcinoma patients with peritoneal dissemination are poor. The biomarker and mechanism causing peritoneal dissemination of gastric cancer cells is still poorly understood.

Fatty acid synthase (FASN) is thought to be a key biosynthetic enzymes in lipogenesis, is regulated by hormones, growth factors and diet. And recent data indicate that it is expressed in several tumor cells. FASN is expressed at low levels in most normal tissues. By contrast, in cancer tissues, the fatty acid supply is highly dependent on de novo biosynthesis via FASN. FASN function and its contribution to gastric cancer development remains poorly understood. It is thought to be a tumor oncogene and promote tumor growth in breast and nasopharyngeal carcinoma (Esslimani-Sahla et al., 2007; Kao et al., 2013; Li et al., 2014). Indeed, several studies have shown that FASN is overexpressed in many cancers (Bandyopadhyay et al., 2005; Ishimura et al., 2011; Uddin et al., 2011; Liu et al., 2012; Kao et al., 2013; Wu et al., 2013). Furthermore, several studies have shown that high FASN expression is associated with advanced clinical stage and poor survival of gastric cancer (Hou et al., 2012). However, researches between FASN and peritoneal dissemination of gastric cancer were relatively infrequent, so the present work was aimed to investigate the relationship between FASN expression and peritoneal dissemination in human gastric cancer.

On the basis of these reports, the aim of the current study was to examine the clinical significance of FASN expression in gastric cancer, and to explore the prognosis and correlation of FASN and peritoneal dissemination 
Hong-Gang Xiang et al

of gastric cancer. We examined FASN expression in gastric carcinoma and paired adjacent normal tissue samples collected from 60 Chinese patients. We analyzed the associations between FASN expression and clini $\neg$ copathological characteristics, such as age, gender, histological grade. We also compared the correlation of expression level of FASN and PTEN in gastric cancer patients. A better understanding of FASN might open a new avenue for targeted therapeutic strategies against peritoneal dissemination of gastric cancer.

\section{Materials and Methods}

\section{Patient population}

Primary tumor samples from 60 patients with histologically confirmed gastric cancer (untreated and with no previous neoadjuvant therapy) who underwent laparotomy for gastrectomy were investigated from the Xinhua Hospital affiliated to Shanghai Jiaotong University School of Medicine. Peritoneal carcinomatosis in diffuse gastric cancer patients was histologically confirmed in 12 of the 60 patients. Formalin-fix, paraffin-embedded tumor tissue blocks were collected. The pathologic reports were reviewed, and the tumor-node-metastasis (TNM) stages were assigned according to the American Joint Committee on Cancer staging system. Ethical approval for this study was granted by the ethics committee of Xinhua hospital and informed consents were obtained.

\section{Immunohistochemical staining}

FASN expression was analyzed using human gastric cancer and normal tissue. Standard immunohistochemical procedures were carried out according to the manufacturer's protocol (Vector Laboratories, USA). Anti-FASN and anti-PTEN monoclonal antibody (Abcam, USA) was used as a primary antibody. Similar tissue sections were immunostained with normal IgG were used as negative controls. The specimens were examined and scored using a two-headed microscope. Staining intensity and the proportion of stained cells were semi-quantitatively determined. The intensity and percentage of positive cell scores were multiplied. All slides were scored by two observers and there are good concordances between two observers. Mean score for duplicate cores from each individual was calculated.

\section{Quantitative RT-PCR}

Human gastric cancer tissues and adjacent normal tissues samples were collected in freezing tubes and were cryopreserved. Total RNA was isolated using Trizol after thawing the samples at room temperature, followed by purification using the RNeasy Mini kit (Qiagen, CA, USA) according to the Manufacturer's instructions. The purity and concentration of total RNA were determined by a NanoDrop ND-1000 spectrophotometer. Quantitative real-time PCR (qRT-PCR) analysis was carried out to detect the expression of FASN. Total RNA was reversetranscribed with Taqman (TakaRa PrimeScript ${ }^{\mathrm{TM}}$ RT Reagent Kit, Japan). Each reaction had a total volume of $20 \mu$, with reagents from the TaKaRa Ex Taq ${ }^{\circledR}$ kit. Data from the array were normalized to GAPDH.

\section{Statistical analysis}

GraphPad Prism version 5.0 for Windows (GraphPad Software, USA) was used for statistics anaylsis. The statistical significance of differences between the various tissues specimens were performed by the $\chi^{2}$ test. Correlation between variables was evaluated by Spearman's rank correlation coefficients. Survival analysis was carried out using the Kaplan-Meier method and logrank test. Differences were considered to be statistically significant at $\mathrm{P}<0.05$.

\section{Results}

Elevated FASN expression in human gastric cancer tissues

The FASN expression in 60 tissue specimens obtained from patients with gastric cancer was assessed by immunohistochemical staining. Positive FASN immunohistochemical staining was predominantly observed in member and cytoplasm of gastric cancer cells (Figure 1a), while weak staining was found in normal gastric tissues. 54 out of 60 specimens $(90 \%)$ of gastric cancer tissues showed positive FASN staining. IHC results demonstrated that higher FASN expression was found in tumor tissues than that in adjacent normal tissues. In this study, FASN expression in 15 fresh tumor specimens from gastric cancer patients was also assessed with the RT-PCR assay. In clinical gastric tissues, FASN mRNA expression was confirmed in most of tumor specimens and higher FASN mRNA expression was found in tumor tissues than that in adjacent normal tissues $(\mathrm{P}<0.01)$ (Figure $1 \mathrm{~b})$.

Correlation between FASN expression and patient's clinicopathological features

To further investigate the clinical relevance of FASN protein levels in the gastric cancer, the correlation between FASN expression and patients' clinicopathologic parameters were analyzed. Our data demonstrated that FASN expression in human gastric cancer tissues was significantly correlated with patient's TNM stage $(\mathrm{P}<0.05)$ (Figure 2a), whereas it is not correlated with patient's age, gender, tumor sizes. It is worth mentioning that higher levels of FASN expression were significantly associated with depth of lymphatic metastasis. There was a trend

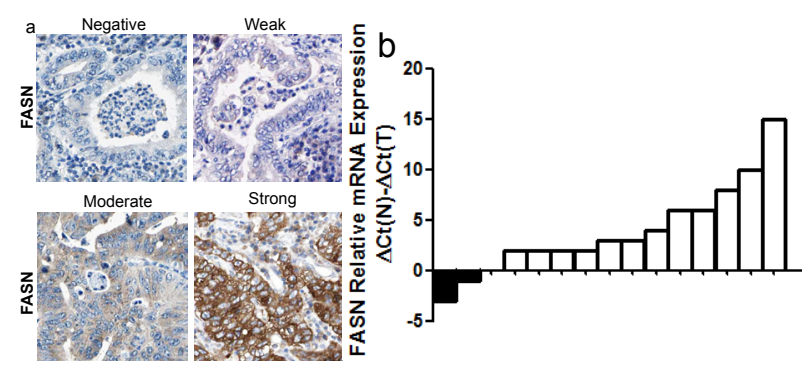

Figure 1. Representative Immunohistochemical Staining of FASN Expression in Gastric Cancer

Tissues. (a) Gastric cancer cells with negative, weak, moderate, and strong expression of FASN. Immunohistochemistry showed that positive FASN immunochemical staining was predominantly observed on the membrane and in cytoplasm of tumor cells. Scale bar, $15 \mu \mathrm{m}$. (b) Quantification of RT-qPCR for FASN mRNA expression in 15 matched human tissues was conducted using paired t test. The relative FASN mRNA expression was standardized to GAPDH 
a

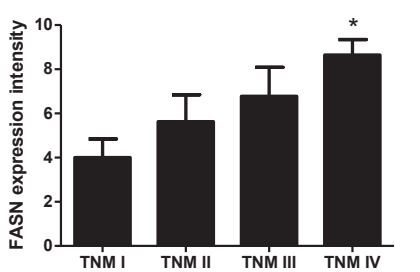

$\mathrm{b}$

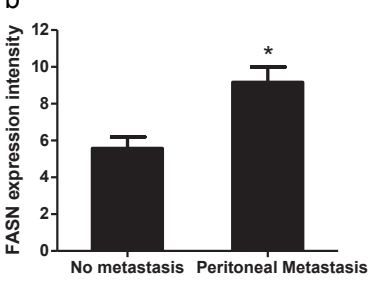

Figure 2. Higher FASN expression associated with TNM stages and peritoneal implanting metastases. (a) FASN is significantly increased in patients with gastric cancer TNM IV compare with TNM I (*,p <0.05). Error bars = s.e.m. (b) FASN was significantly increased in gastric cancer patients with peritoneal dissemenation compared with non-metastasis gastric cancer $(*, p<0.05)$. Error bars $=$ s.e.m

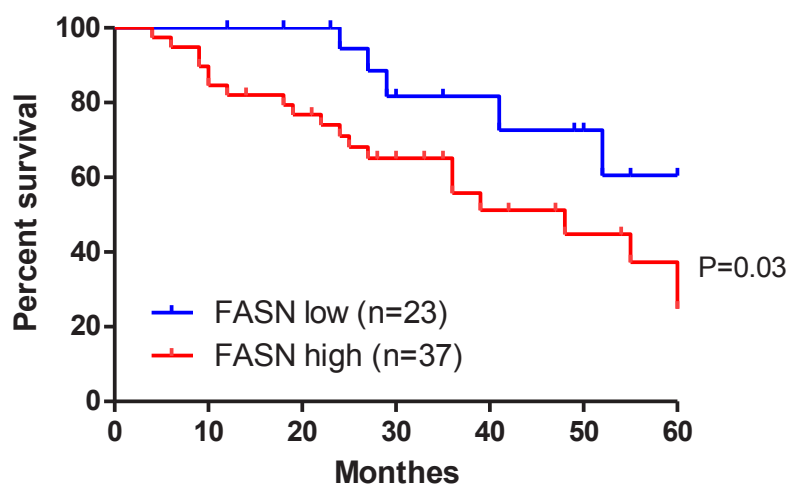

Figure 3. The Kaplan-Meier Survival Curves for Gastric Cancer Patients Based on FASN Expression. FASN Patients with high FASN expression (Score $>6$ ) had a significantly poorer prognosis than those with low FASN expression $(\mathrm{p}=0.03)$
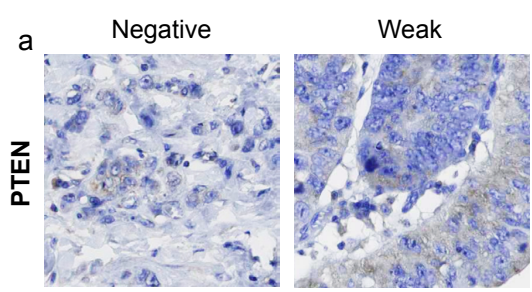

b

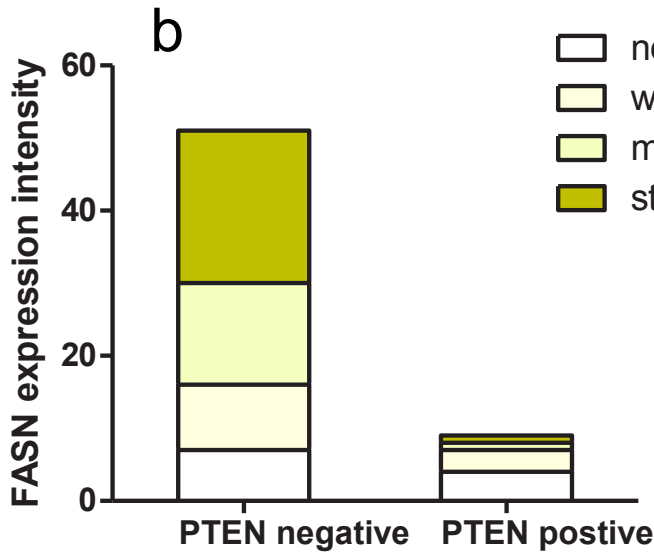

Figure 4. The Correlation between FASN Expression Status and PTEN Expression in Gastric Cancer. (a) Representative immunohistochemical staining for the PTEN expression (negative, weak and moderate) in gastric cancer. Scale bar, $15 \mu \mathrm{m}$. (b) The FASN expression status was higher in gastric cancer patients with negative PTEN expression $(\mathrm{P}<0.01)$ toward a positive correlation between FASN expression and metastasis. Thus, our data supported that FASN was involved in the progression of human gastric cancer.

Higher FASN expression associated with Peritoneal Implanting Metastases and poor prognosis in human gastric cancer

Elevated FASN was associated with gastric cancer progression, which reminded me to explore the association of FASN and peritoneal dissemination. 12 of the 60 patients in our cohort were confirmed to peritoneal metastasis, and higher FASN expression was found in peritoneal dissemination patients than that those without peritoneal dissemination (Table 1) (Figure 2b). FASN might play a crucial role in the establishment of peritoneal dissemination. We then evaluated the association between FASN expression and survival following gastrectomy in gastric cancer patients. Logistic regression analysis revealed that elevated FASN expression was an independent risk factor for survival (Figure 3 ). The survival rates were significantly lower in patients with high FASN expression than in those with low FASN expression $(\mathrm{P}<0.05)$ (Table 2$)$. Our studies support the utility of FASN as prognostic biomarkers for peritoneal dissemination and prognosis biomarker in gastric cancer patients undergoing gastrectomy.

FASN expression negatively correlated with PTEN expression in gastric cancer

To investigate the potential mechanism of FASN on gastric cancer progression, we investigated the relationship between FASN expression and Phosphatase and tensin homolog (PTEN). PTEN is a tumor suppressor involved in multiple cell processes (Chen et al., 2014; $\mathrm{Xu}$ et al., 2014). The loss or reduced expression of PTEN potentially correlate with advanced stages of gastric carcinoma (Zhu et al., 2013), and PTEN has been identification of peritoneal metastasis-related genes of gastric adenocarcinoma (Bai et al., 2012). PTEN expression was identified in our gastric cancer tissues (Figure 4a), and the results showed that PTEN expression was significantly lower in gastric cancer patients with high FASN expression than those in patients with low FASN expression (Table 1). Logistic regression analysis revealed that decreased PTEN expression was an independent risk factor for survival (Table 2). Consequently, the FASN expression status of gastric cancer cells was inversely correlated with the PTEN expression $(\mathrm{P}<0.01)$ (Figure

Table 1. Relationship between FASN and PTEN Expression in Gastric Cancer

\begin{tabular}{lrrrr}
\hline & \multicolumn{3}{c}{ FASN expression } & P value \\
\cline { 2 - 3 } & All & $\begin{array}{c}\text { High } \\
(\text { score }>6)\end{array}$ & $\begin{array}{c}\text { Low } \\
(\text { score } \leq 6)\end{array}$ & \\
\hline PTEN expression & & & \\
Negative & 51 & $35(69 \%)$ & $16(31 \%)$ & \\
Positive & 9 & $2(22 \%)$ & $7(78 \%)$ & 0.02 \\
Peritoneal dissemination status & & \\
Negative & 48 & $26(54 \%)$ & $22(46 \%)$ & \\
Positive & 12 & $11(92 \%)$ & $1(8 \%)$ & 0.02 \\
\hline
\end{tabular}


Table 2. Multivariate Cox proportional hazards regression for metastasis-free survival of Patients with Gastric Cancer

\begin{tabular}{lcc}
\hline Variables & $\begin{array}{c}\text { Multivariate HR } \\
(95 \% \text { CI })\end{array}$ & P value \\
\hline Gender & $0.53(0.17-1.51)$ & 0.41 \\
Age & $0.92(0.89-1.02)$ & 0.19 \\
Tumor stage & $0.29(0.09-1.20)$ & 0.05 \\
Lymph node metastasis & $0.96(0.54-1.88)$ & 0.03 \\
Peritoneal Dissemination & $1.93(1.52-2.28)$ & 0.01 \\
FASN expression & $1.44(1.08-1.91)$ & 0.02 \\
PTEN expression & $1.10(0.92-1.75)$ & 0.04 \\
\hline
\end{tabular}

*HR, hazard ratio; CI, confidence interval

4b). Elevated FASN expression and loss of PTEN maybe involved the peritoneal dissemination of gastric cancer.

\section{Discussion}

In the present study, FASN expression was examined in patients with gastric cancer. As aforementioned, we demonstrated that higher FASN expression was positively correlated with advanced TNM stage and poor patient outcome, implicating its role in gastric cancer progression. Moreover, high FASN expression in gastric cancer tissues correlates with peritoneal metastasis, which demonstrated FASN might play a crucial role in the establishment of peritoneal dissemination. FASN expression was inversely correlated with the expression of PTEN, and both account for the progression and peritoneal dissemination of gastric cancer. We believe that, with further investigation, FASN could become a clinical prognostic marker and a target for therapeutic treatment of human gastric cancer.

During malignant transformation, de novo lipogenesis account for the fatty acids in malignant cells due to increased endogenous lipid biosynthesis (Furuta et al., 2010). FASN, the main enzyme involved in fatty acids synthesis, have been demonstrated to be upregulated in different tumors, which may contribute to tumor immune evasion (Zaytseva et al., 2012; Zaytseva et al., 2014). Accumulated evidences has been shown that increased FASN expression is involved in shaping the tumor microenvironment, and aberrant FASN expression is associated with various clinicopathological features in many human malignancies (Vazquez-Martin et al., 2008; Migita et al., 2009; Vazquez-Martin et al., 2009; Flavin et al., 2010; Rahman et al., 2012). FASN significantly correlated with depth of tumor invasion, lymph node metastasis, and overall stage in gastric cancer. FASN expression was significantly higher in invasive breast cancer cells and increased FASN expression was associated with negative progesterone receptor status in breast cancer patients. Furthermore, the measurement of FASN expression is expected to become a useful tool for the prediction of radiation therapeutic response and prognosis in nasopharyngeal cancer patients (Kao et al., 2013). In prostate cancer, FASN act as a prostate cancer oncogene and exerts its oncogenic effect by inhibiting the intrinsic pathway of apoptosis (Migita et al., 2009). In the present study, primary gastric tumor cells displayed various degrees of FASN expression, and its expression was observed in $90 \%$ patients with gastric cancer. These results indicate that the majority of patients with gastric cancer express FASN and that it may be a useful diagnostic marker for patients with gastric cancer.

In this study, we showed that FASN expression in human gastric cancer tissues was significantly correlated with patient's TNM stage and extragastrical extension. Moreover, gastric cancer patients with high FASN expression tended to display the presence of peritoneal metastasis compared with those with low FASN expression. Our findings indicated a close relationship between FASN expression and tumor progression in gastric cancer. Furthermore, patients with high FASN expression had a poorer prognosis compared with those with low FASN expression in human gastric cancer. Our studies support the utility of FASN as prognostic biomarkers in gastric cancer patients undergoing gastrectomy. To date, the functional role of FASN expression in gastric cancer cells remains unclear and the mechanisms for FASN-mediated cancer cell malignance are not clear yet. It has been shown that the activation of the FASN signaling pathway may lead to tumor cells epithelial-mesenchymal transition $(\mathrm{Li}$ et al., 2014). Several evidences have demonstrated that the FASN promotes cancer metastasis via CD44-mediated the activation of MET, Akt and FAK, which are known to regulate adhesion, migration, and invasion (Zaytseva et al., 2012). In this study, our data demonstrated that loss of PTEN correlated with increased FASN in gastric cancer. Decreased PTEN expression has been shown to be correlated with the progressive outcome of solid cancers, including breast and colorectal cancer (Yoshimoto et al., 2007; Molinari and Frattini, 2013). PTEN has been also shown to be a tumor suppressor of gastric cancer, which involved the tumor growth and metastasis of gastric cancer (Zhu et al., 2013; Xu et al., 2014). Combining with our results, the relationship of FASN and PTEN maybe account for the progression and peritoneal dissemination of gastric cancer. It is clear that further investigations are necessary to fully understand the mechanistic and functional role of FASN, and larger patient populations are need to determine whether FASN expression has a role in predicting patient outcome in gastric cancer.

In conclusions, our results demonstrate that FASN is involved in gastric cancer progression and peritoneal metastasis and could be a useful prognostic indicator for human gastric cancer. Future studies on the biological behavior of gastric cancer cells expressing FASN may lead to a new therapy blocking its signaling pathway in gastric cancer patients with peritoneal dissemination.

\section{Acknowledgements}

We are grateful to all staffs of department of surgery in Xinhua hospital for cooperating with us in the sample collection. The authors have no direct or indirect commercial financial incentive associated with publishing the article.

\section{References}

Ab ai FH, Wang NJ, Wang J, et al (2012). Screening and 
Fatty Acid Synthase Expression Negatively Correlates with PTEN and Predicts Peritoneal Dissemination of Gastric Cancer

identification of peritoneal metastasis-related genes of gastric adenocarcinoma using a cDNA microarray. Genet Mol Res, 11, 1682-9.

Bandyopadhyay S, Pai SK, Watabe M, et al (2005). FAS expression inversely correlates with PTEN level in prostate cancer and a PI 3-kinase inhibitor synergizes with FAS siRNA to induce apoptosis. Oncogene, 24, 5389-95.

Chen J, Li T, Liu Q, et al (2014). Clinical and prognostic significance of HIF-1alpha, PTEN, CD44v6, and survivin for gastric cancer: a meta-analysis. PLoS One, 9, e91842.

Esslimani-Sahla M, Thezenas S, Simony-Lafontaine J, et al (2007). Increased expression of fatty acid synthase and progesterone receptor in early steps of human mammary carcinogenesis. Int J Cancer, 120, 224-9.

Flavin R, Peluso S, Nguyen PL, et al (2010). Fatty acid synthase as a potential therapeutic target in cancer. Future Oncol, 6, $551-62$.

Furuta E, Okuda H, Kobayashi A, et al (2010). Metabolic genes in cancer: their roles in tumor progression and clinical implications. Biochim Biophys Acta, 1805, 141-52.

Hou W, Fei M, Qin X, et al (2012). High overexpression of fatty acid synthase is associated with poor survival in Chinese patients with gastric carcinoma. Exp Ther Med, 4, 999-1004.

Ishimura N, Amano Y, Sanchez-Siles AA, et al (2011). Fatty acid synthase expression in Barrett's esophagus: implications for carcinogenesis. J Clin Gastroenterol, 45, 665-72.

Kao YC, Lee SW, Lin LC, et al (2013). Fatty acid synthase overexpression confers an independent prognosticator and associates with radiation resistance in nasopharyngeal carcinoma. Tumour Biol, 34, 759-68.

Li JQ, Xue H, Zhou L, et al (2014). Mechanism of fatty acid synthase in drug tolerance related to epithelial-mesenchymal transition of breast cancer. Asian Pac J Cancer Prev, 15, 7617-23.

Liu ZL, Wang G, Peng AF, et al (2012). Fatty acid synthase expression in osteosarcoma and its correlation with pulmonary metastasis. Oncol Lett, 4, 878-82.

Migita T, Ruiz S, Fornari A, et al (2009). Fatty acid synthase: a metabolic enzyme and candidate oncogene in prostate cancer. J Natl Cancer Inst, 101, 519-32.

Molinari F, Frattini M (2013). Functions and Regulation of the PTEN Gene in Colorectal Cancer. Front Oncol, 3, 326.

Rahman MT, Nakayama K, Rahman M, et al (2012). Fatty acid synthase expression associated with NAC1 is a potential therapeutic target in ovarian clear cell carcinomas. $\mathrm{Br} J$ Cancer, 107, 300-7.

Uddin S, Jehan Z, Ahmed M, et al (2011). Overexpression of fatty acid synthase in Middle Eastern epithelial ovarian carcinoma activates AKT and Its inhibition potentiates cisplatin-induced apoptosis. Mol Med, 17, 635-45.

Vazquez-Martin A, Colomer R, Brunet J, et al (2008). Overexpression of fatty acid synthase gene activates HER $1 /$ HER2 tyrosine kinase receptors in human breast epithelial cells. Cell Prolif, 41, 59-85.

Vazquez-Martin A, Fernandez-Real JM, Oliveras-Ferraros C, et al (2009). Fatty acid synthase activity regulates HER2 extracellular domain shedding into the circulation of HER2positive metastatic breast cancer patients. Int J Oncol, 35, 1369-76.

Wu D, Xu J, Yu G, et al (2013). Expression Staus of Fatty Acid Synthase (FAS) but not HER2 is Correlated with the Differentiation Grade and Prognosis of Esophageal Carcinoma. Hepatogastroenterol, 60, 99-106.

Xu WT, Yang Z, Lu NH (2014). Roles of PTEN (Phosphatase and Tensin Homolog) in gastric cancer development and progression. Asian Pac J Cancer Prev, 15, 17-24.

Yoshimoto M, Cunha IW, Coudry RA, et al (2007). FISH analysis of 107 prostate cancers shows that PTEN genomic deletion is associated with poor clinical outcome. Br J Cancer, $\mathbf{9 7}$, 678-85.

Zaytseva YY, Elliott VA, Rychahou P, et al (2014). Cancer cellassociated fatty acid synthase activates endothelial cells and promotes angiogenesis in colorectal cancer. Carcinogenesis., 35, 1341-51.

Zaytseva YY, Rychahou PG, Gulhati P, et al (2012). Inhibition of fatty acid synthase attenuates CD44-associated signaling and reduces metastasis in colorectal cancer. Cancer Res, 72, 1504-17.

Zeng H, Zheng R, Guo Y, et al (2014). Cancer survival in China, 2003-2005: A population-based study. Int J Cancer, 136, 1921-30.

Zhu X, Qin X, Fei M, et al (2013). Loss and reduced expression of PTEN correlate with advanced-stage gastric carcinoma. Exp Ther Med, 5, 57-64. 